\title{
Systemic Administration of an Antagomir Designed to Inhibit miR-92, a Regulator of Angiogenesis, Failed to Modulate Skeletal Anabolic Response to Mechanical Loading
}

\author{
A. SENGUL ${ }^{1}$, R. SANTISUK ${ }^{1}$, W. XING ${ }^{1,2}$, C. KESAVAN $^{1,2}$ \\ ${ }^{1}$ Musculoskeletal Disease Center, JLP VA Medical Center, Loma Linda, CA, USA, ${ }^{2}$ Department of \\ Medicine, Loma Linda University, Loma Linda, CA, USA
}

Received June 15, 2012

Accepted September 21, 2012

On-line December 13, 2012

\section{Summary}

The goal of this study is to evaluate if promotion of angiogenesis by systemic treatment with an antagomir against miR-92a, a well established inhibitor of angiogenesis, will maximize the benefits of exercise on bone. Ten week old female C57BL6/J mice were subjected to two weeks of external load by four point bending. During the first week of mechanical loading (ML), mice were injected $(2.7 \mathrm{mg} / \mathrm{kg}$ of bodyweight) with antagomir against miR-92 or control antagomir (3 alternate days via retro-orbital). No difference in tissues weights (heart, kidney, liver) were found in mice treated with miR-92 vs. control antagomir suggesting no side effects. Two weeks of ML increased tibia TV, BV/TV and density by $6-15 \%$, as expected, in the control antagomir treated mice. Similar increases in the above parameters (7-16 \%) were also seen in mice treated miR-92 antagomir. Administration of miR-92 antagomir was effective in reducing levels of mir-92 in heart, liver and skeletal muscle and in contrast, expression levels of two other microRNA's miR-93 and miR-20a remain constant, thus suggesting specificity of the antagomir used. Surprisingly, we failed to detect significant changes in the expression levels of vascular genes (VEGF, CD31 and Tie2) in heart, liver or skeletal muscle. Based on these findings, we conclude that systemic administration of antagomir against miR-92 while reduced expression levels of miR-92 in the tissues; it did not significantly alter either angiogenic or osteogenic response, thus suggesting possible redundancy in miR-92 regulation of angiogenesis.

\section{Key words}

microRNA - Mechanical loading • Angiogenesis • Mice • microRNA-92

\section{Corresponding author}

C. Kesavan, Musculoskeletal Disease Center (151), Jerry L. Pettis Memorial VA Medical Center, 11201 Benton Street, Loma Linda, CA 92357, USA. Fax: 909-796-1680. E-mail: Chandrasekhar.Kesavan@va.gov

Bone is a highly vascularized tissue that reliant on the close spatial and temporal connection between blood vessels and bone cells to maintain skeletal integrity. Several experimental studies have shown that angiogenesis plays a vital role in skeletal development, repairing fractured bone and in response to mechanical loading (ML). These includes 1) Studies using a distraction osteogenesis model (DO) have shown that intramembranous bone formation is induced by the application of gradual mechanical distraction across an osteotomy defect, which reveals not only an increase in osteogenesis but also an increase in expression of several angiogenic factors (Carvalho et al. 2004). 2) Treadmillrunning in rats displayed bone marrow angiogenesis concomitant with increase in osteogenesis (Yao et al. 2004). 3) Studies using mandibular DO model have shown that high frequency traction provides a proper mechanical environment for angiogenesis contributing to enhanced bone formation. These findings illustrate that angiogenesis, osteogenesis and ML are tightly related (Zheng et al. 2009).

Recently, newly discovered microRNA's belonging to a small class of RNA molecules have 
received considerable attention because of the ability to act as negative regulators of gene expression. RNA of this type regulates gene expression at the posttranscriptional level by either degradation or translational repression of a target mRNA. So far, at least 500 microRNA's have been discovered of which few are linked to pathogenesis of disease (heart disease, cancer and schizophrenia) as evident from human and animal studies (Bravo and Dinan 2010, Abdellatif 2012, Zhang and Ma 2012). In particular, in vitro and in vivo models have demonstrated that over expression of miR-92 blocked angiogenesis in human endothelial cells and that blocking miR-92 recovered mouse from limb ischemia injury as well as from myocardial infarction, respectively (Bonauer et al. 2009). Since angiogenesis and osteogenesis tightly coupled, we predict that inhibiting microRNA-92 that control angiogenesis can maximize the benefits of exercise on skeleton. Based on this and the above rationale, we propose the hypothesis that blocking microRNA that control angiogenesis will increase the magnitude of anabolic effects of ML on bone formation.

To test the above hypothesis, 9 week old female C57BL/6J mice were purchased from Jackson Laboratory. All the mice were housed under the standard conditions of 14-h light and 10-h darkness, and had free access to food and water. The experimental protocols were in compliance with animal welfare regulations and approved by local IACUC. To establish a loading model that show maximum change in vascularization and osteogenesis, we performed ML using four-point bending and axial method of loading on a 10 week old female C57BL/6J (B6) mice (Kesavan et al. 2005, 2011, Sengul et al. 2012). In the four-point bending model, a $9 \mathrm{~N}$ load was applied on the right tibia of B6 mice over the muscle and soft tissue at $2 \mathrm{~Hz}$ frequency, 36 cycles once per day under inhalable anesthesia (5\% Isoflurane and $95 \%$ oxygen) for a period of 2 weeks ( 6 days/week with 1 day rest). The left tibia was used as contra-lateral internal control. We used 9N load for the four-point bending method of loading since in the past, we have shown that $9 \mathrm{~N}$ load produce robust bone response than other loads (Kesavan et al. 2005). Also, based on our past experience working with this model, we found that load around $12 \mathrm{~N}$ or more induces fractures in the four-point bending method of loading. On day 15, mice were euthanized and tibias were collected for further study. Similarly, in the axial loading model, a $10 \mathrm{~N}$ load was applied on the right tibia of B6 mice and the left tibia was used as nonexternally loaded control. The loading was performed for a period of 2 weeks ( 3 alternate days, 40 cycles with $10 \mathrm{~s}$ rest). On day 15, mice were euthanized and tibias were collected for RNA extraction. In our study, we used Trizol followed by Direct-zol kit (Zymo Research, USA) to extract RNA from marrow flushed bones and tissue samples. Quality and quantity of RNA were analyzed using a 2100 Bio-analyzer (Agilent, Palo Alto, CA, USA) and Nano-drop (Wilmington, DE). To quantitate expression levels of bone formation genes and vascular genes, briefly, purified total RNA [200 $\mu \mathrm{g} / \mu \mathrm{l}]$ was used to synthesize the first strand cDNA by reverse transcription according to the manufacturer's instructions [Bio-Rad, Hercules, CA USA]. $5 \mu$ l of the five times diluted first strand cDNA reaction, was subjected to real time PCR amplification using gene specific primers as described earlier (Kesavan et al. 2011). The gene specific primers for bone formation (DMP1, Osterix, EphB2 and SDF1) and angiogenesis (CD 31, Tie 2) were designed by using Vector NTI software and ordered from IDT DNA technologies (USA). Approximately $20 \mu \mathrm{l}$ of reaction volume was used for the real time PCR assay that consisted of $1 \mathrm{X}[10 \mu \mathrm{l}]$ Universal SYBR green PCR master mix [Master mix consists of SYBR Green dye, reaction buffers, dNTPs mix, and Hot Start Taq polymerase] [Applied Biosystems, Foster City, CA], $50 \mathrm{nM}$ of primers, $15 \mu \mathrm{l}$ of water, and $5 \mu \mathrm{l}$ of template. The thermal conditions consisted of an initial denaturation at $95^{\circ} \mathrm{C}$ for $10 \mathrm{~min}$ followed by 40 cycles of denaturation at $95{ }^{\circ} \mathrm{C}$ for $15 \mathrm{~s}$, annealing and extension at $60{ }^{\circ} \mathrm{C}$ for $1 \mathrm{~min}$, and a final step melting curve of $95^{\circ} \mathrm{C}$ for $15 \mathrm{~s}, 60^{\circ} \mathrm{C}$ for $15 \mathrm{~s}$, and $95^{\circ} \mathrm{C}$ for $15 \mathrm{~s}$. The data were analyzed using SDS software, version 2.0, and the results were exported to Microsoft Excel for further analysis. Data normalization was accomplished using the endogenous control $[\beta$-actin] and the normalized values were subjected to a $2^{-\Delta \Delta} \mathrm{Ct}$ formula to calculate the fold change between the loaded and non-loaded tibias.

To block miR-92, a microRNA that is well known in regulating angiogenesis, single stranded RNA antagomir sequences against miR-92 and control antagomir sequence were ordered from IDT DNA technology. The sequences were obtained from previously published manuscript (Bonauer et al. 2009). In the miR-92 antagomir and control antagomir, the 2'O RNA base are methylated followed by first two bases and last 3 bases are phosphorothiated to increase the stability of antagomir from degradation. In addition, a cholestrol-TEG was added at the 3' for easy entry of antagomir into the cells. Antagomir for miR-92a 
(5 $\left.{ }^{`}-\mathrm{CAGGCCGGGACAAGUGCAAUA-3}{ }^{\prime}\right)$ and Antagomir-Co (5`-AAGGCAAGCUGACCCUGAA GUU-3 ${ }^{`}$ ). To test if blocking miR-92 maximizes the benefit of exercise on bone, we performed ML using four-point bending on 10 week old female B6 mice for a period of 2 weeks. The loading regimens are mentioned above. During the first week of loading, mice were injected with antagomir against miR-92 and control antagomir via retro-orbital under $5 \%$ Isoflurane and $95 \%$ oxygen anesthesia for 3 alternate days $(2.7 \mathrm{mg} / \mathrm{kg}$ of body weight). Two weeks after the last day of loading mice were euthanized and tibias were collected to evaluate if there is an increase or decrease in skeletal parameters using micro-CT (Scanco Invivo CT40, Switzerland) as described earlier on the cortical bone site (Kesavan et al. 2011). Furthermore, to assure that the antagomir delivered into mouse model blocked miR-92, expression levels of miR-92, U6 (internal control) and two other microRNA's (miR-93 and miR-20a) as a positive control were quantitated in liver, skeletal muscle and kidney of mir-92 antagomir and control antagomir treated mice. Levels of microRNA's were evaluated using microRNA specific stem-loop RT primer and by Real time PCR primer that were obtained from Applied Biosystem and reactions were performed according to the manufacture instructions. In brief, $10 \mathrm{ng}$ of RNA was used synthesize the first strand of cDNA by reverse transcription using respective stem-loop microRNA RT primer. Approximately $10 \mu \mathrm{l}$ of reaction volume was used for the RT assay that consisted of $0.1 \mu \mathrm{l}$ of 100 dNTPs, $1 \mu 1$ of 10X Buffer, $0.5 \mu 1$ of reverse transcriptase enzyme, $0.12 \mu \mathrm{l}$ of $50 \mathrm{U} / \mu \mathrm{l}$ RNasin, $1 \mu \mathrm{l}$ of $5 \mathrm{X}$ RT primer, $6.78 \mu \mathrm{l}$ of Nuclease free water and $0.5 \mu 1$ of $10 \mathrm{ng}$ RNA. $5 \mu 1$ of the five times diluted first strand cDNA reaction was subjected to real time PCR amplification using microRNA specific real time PCR primers. Approximately $20 \mu \mathrm{l}$ of reaction volume was used for the assay that consisted of $10 \mu \mathrm{l}$ of TaqMan 2X Universal PCR Master mix, no AmpErase UNG, $0.5 \mu 1$ of $20 \mathrm{X}$ probe against target microRNA and $4.5 \mu 1$ of Nuclease free water, and $5 \mu \mathrm{l}$ of DNA. The data were analyzed using SDS software, version 2.0, and the results were exported to Microsoft Excel for further analysis. Data normalization was accomplished using U6 as internal control and the normalized values were subjected to a $2^{-\Delta \Lambda} \mathrm{Ct}$ formula to calculate the fold change between the antagomir and control antagomir treated groups.

The data presented are given as mean $\pm \mathrm{SD}$.
Standard t-test was used to compare the difference between externally loaded versus non-loaded bones. We used Statistica software (StatSoft, Inc version 7.1, 2005) to perform the analysis and the results were considered significant at $\mathrm{p}<0.05$.

To date, several in vivo loading models have been used to stimulate new bone formation in mouse models. Among these loading models, we selected fourpoint bending and axial method of loading for two main reasons: 1) the amount of load, cycle and frequency applied on mouse tibia is controlled in both models. 2) Two- weeks of loading caused a robust increase in new bone formation in both models. Therefore, we used ML models to test our hypothesis that ML induced increase in angiogenesis is coupled to new bone formation at the loaded site using a 10 week old B6 mice. The rationale for selecting 10 week mice and not young mice for the study is that growing mice have high bone formation rate. Therefore, performing mechanical loading in combination with antagomir treatment in growing mice will reduce the possibility of capturing loading induced bone response difference between groups. Furthermore, in our study, we used CD31 and Tie 2 genes to evaluate angiogenesis. Reports have shown that these two genes are highly expressed on the surface of the endothelial cells in site of neo-vascularization or vascularization and an increase in the expression levels of these genes corresponds to an increase in vascularization (Peters et al. 1998, Sapino et al. 2001, Galeano et al. 2004). Therefore, we chose these two genes to determine if loading induces an increase in angiogenesis in bone. As shown in Figure 1, 2 weeks of four-point bending and axial method of loading induced significant changes in the expression levels of bone formation markers as well as angiogenic markers genes. Furthermore, positive correlations between vascular and osteogenic genes $(r=0.30-0.83$, $\mathrm{p}<0.05, \mathrm{~N}=6-8$ ) suggests that loading induced increase in bone response is in part associated with an increase in vascularization. Although both loading models induced similar response in both vascularization and angiogenesis, we chose four-point bending of loading for further study because this method of loading causes a more robust increase in periosteal bone response in the form of woven bone at the loaded site compared to axial loading and therefore provided as opportunity to evaluate the consequence of blockade of angiogenic response on ML induced periosteal bone formation. 
(1)

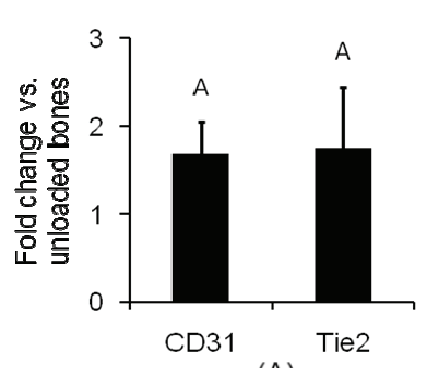

(A)

(2)

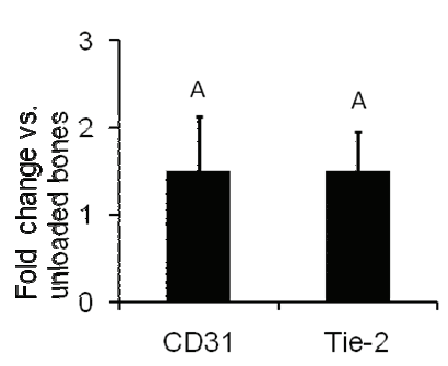

(A)

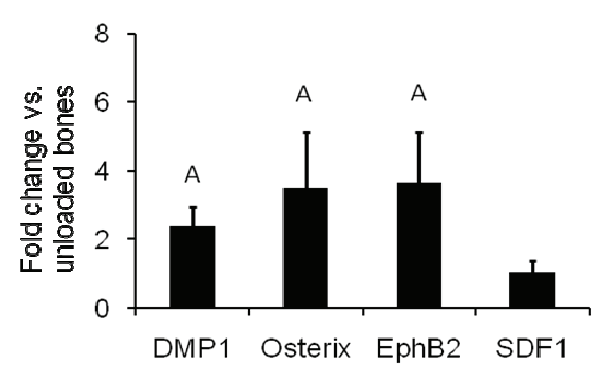

(B)

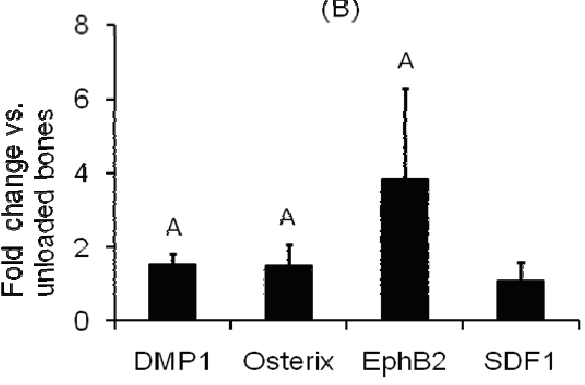

Fig. 1. Quantization of mRNA levels of (A) vascular and (B) osteogenic markers after 2 weeks of (1) four-point bending and (2) axial method of loading in female B6 mice. The $y$-axis corresponds to fold change and $\mathrm{x}$-axis represents genes. Values are mean $\pm S D, N=7-8$. ${ }^{A} p<0.05$ vs. unloaded bones.

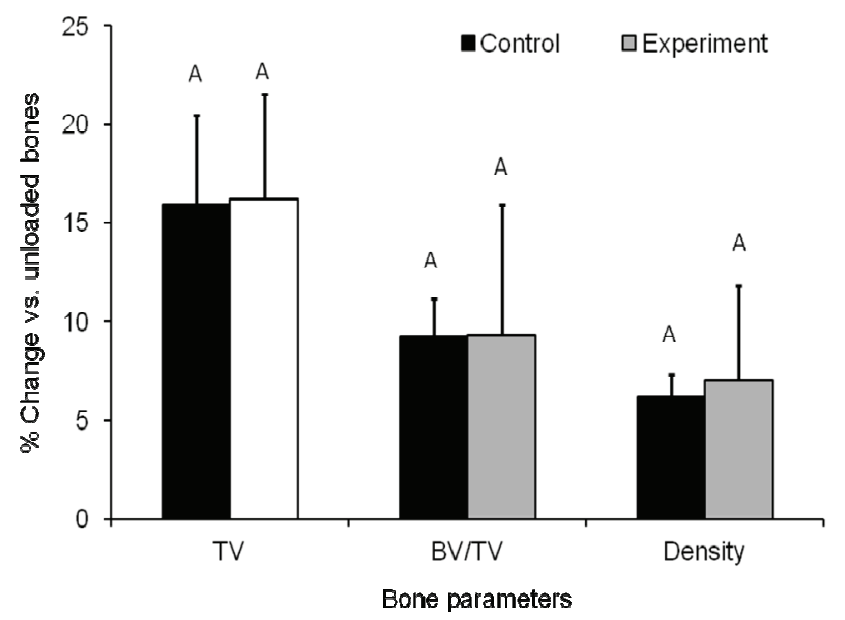

Fig. 2. Micro-CT measurements of bone parameters (diaphysis area) after 2 weeks of loading in female B6 mice treated with control antagomir and antagomir against miR-92. The $y$-axis corresponds to percent change and $\mathrm{x}$-axis represents bone parameters. TV: Tissue volume, BV/TV: Bone volume/Tissue volume and density: total bone density. Values are mean $\pm S D$, $\mathrm{N}=4 .{ }^{A} \mathrm{p}<0.05$ vs. unloaded bones.

Several studies have used antagomir to block target microRNA at a concentration ranging from $0.33 \mathrm{mg}$ to $100 \mathrm{mg} / \mathrm{kg}$ of bodyweight in animal models. The issue of whether the doses used are optimal and specific to target microRNA has not been examined thoroughly. However, one potential concern with the use of high dose of antagomir is that antagomir at high concentrations could produce non-specific effects by inhibiting other genes besides target. Since reports have shown $0.33-1.0-$ and $3.3-\mathrm{mg} / \mathrm{kg}$ of dosage was effective in exhibiting the biological response in tissues (Hullinger et al. 2012), we chose lower dosage of antagomir for the study ( $2.7 \mathrm{mg} / \mathrm{kg}$ of bodyweight). To determine whether blocking miR-92 will maximize the benefits of exercise on bone via increasing angiogenesis, we injected antagomir against miR-92 and control antagomir via retro-orbital approach for a period of one week (3 alternate days) while the mice were subjected to loading regimens. After two weeks of loading, there was no significant difference in the body weight between control and experiment group $(19.6 \pm 0.1 \mathrm{~g}$ vs. $19.7 \pm$ $0.26 \mathrm{~g}$ ). Similarly, we did not see any difference in the tissue weights in liver $(0.88 \pm 0.02$ vs. $0.83 \pm 0.04)$, kidney $(0.10 \pm 0.006$ vs. $0.10 \pm 0.005)$ and heart $(0.10 \pm$ 0.006 vs. $0.11 \pm 0.010)$ between the control and experimental groups suggesting that there is no side effects caused by the antagomir or control antagomir injections.

Micro-CT analysis revealed that two weeks of ML increased tibia tissue volume (TV), bone volume/tissue volume (BV/TV) and bone density by $6-15 \%$, as expected, in the control antagomir treated mice. Surprisingly, similar increases in TV (16\%), BV/TV (9\%) and bone density (7\%) were also seen in mice treated with miR-92 antagomir (Fig. 2) and because of this reason; we did not perform any histomorphometric analysis on the bone samples treated with antagomir against mir-92 and control antagomir. Since there was no difference between the groups, this, then raises a question whether systemic administration of mir-92 antagomir was effective in reducing the levels of miR-92. To test this, real time RT-PCR was performed in RNA samples of heart and skeletal muscle for miR-92 and two-other microRNA's to determine specificity. The results from our study revealed a 5-14 fold decrease in miR-92 levels 
in the heart, liver and skeletal muscles of mice treated with miR-92 antagomir compared to control antagomir. In contrast, expression levels of two other microRNA's miR-93 and -20a were not different between the two groups of mice, thus suggesting specificity of the antagomir used. Since past reports have shown that mir-92 is important regulator of angiogenesis, we also determined whether blocking miR-92 maximized the expression levels of vascular marker genes in the above tissues samples. Surprisingly, we failed to detect significant changes in the expression levels of vascular genes (VEGF, CD31 and Tie2) in heart liver or skeletal muscle at the time points examined. We offer the following potential explanations for the negative results in this study: 1) the dosage of antagomir used in our study was slightly less compared to earlier study ( $2.7 \mathrm{mg} / \mathrm{kg}$ of body weight vs. $8 \mathrm{mg} / \mathrm{kg}$ of body weight) (Bonauer et al. 2009). The issue of whether higher dosage is required to see a change in the phenotype or whether the inhibitory effect on angiogenesis seen in the previous study was due to secondary effects on other genes require further evaluation. 2) The expression levels of angiogenic genes were examined only at one time point and additional time points may need to be examined. 3) Earlier study measured an increase in angiogenesis by blood flow while we examined expression levels of vascular genes.

Based on our above findings, we conclude that systemic administration of antagomir against miR-92, while it reduced expression levels of miR-92 in the skeletal muscle, liver and heart; it did not significantly alter either angiogenic or osteogenic response, thus suggesting possible redundancy in miR-92 regulation of angiogenesis.

\section{Conflict of Interest}

There is no conflict of interest.

\section{Acknowledgements}

This work was supported by the Department of Defense Army, Award No W81XWH-10-1-0718. The US Army Medical Research Acquisition Activity (Fort Detrick, MD) 21702-5014 is the awarding and administering acquisition office for the DAMD award. The information contained in this publication does not necessarily reflect the position or the policy of the Government, and no official endorsement should be inferred. All work was performed in facilities provided by the Department of Veterans Affairs.

\section{References}

ABDELLATIF M: Differential expression of microRNAs in different disease states. Circ Res 110: 638-650, 2012.

BONAUER A, CARMONA G, IWASAKI M, MIONE M, KOYANAGI M, FISCHER A, BURCHFIELD J, FOX H, DOEBELE C, OHTANI K, CHAVAKIS E, POTENTE M, TJWA M, URBICH C, ZEIHER AM, DIMMELER

S: MicroRNA-92a controls angiogenesis and functional recovery of ischemic tissues in mice. Science 324: 1710-1713, 2009.

BRAVO JA, DINAN TG: MicroRNAs: a novel therapeutic target for schizophrenia. Curr Pharm Des 17: 176-188, 2010.

CARVALHO RS, EINHORN TA, LEHMANN W, EDGAR C, AL-YAMANI A, APAZIDIS A, PACICCA D, CLEMENS TL, GERSTENFELD LC: The role of angiogenesis in a murine tibial model of distraction osteogenesis. Bone 34: 849-861, 2004.

GALEANO M, ALTAVILlA D, CUCINOTTA D, RUSSO GT, CALO M, BITTO A, MARINI H, MARINI R, ADAMO EB, SEMINARA P, MINUTOLI L, TORRE V, SQUADRITO F: Recombinant human erythropoietin stimulates angiogenesis and wound healing in the genetically diabetic mouse. Diabetes 53: 2509-2517, 2004.

HULLINGER TG, MONTGOMERY RL, SETO AG, DICKINSON BA, SEMUS HM, LYNCH JM, DALBY CM, ROBINSON K, STACK C, LATIMER PA, HARE JM, OLSON EN, VAN ROOIJ E: Inhibition of miR-15 protects against cardiac ischemic injury. Circ Res 110: 71-81, 2012.

KESAVAN C, MOHAN S, OBERHOLTZER S, WERGEDAL JE, BAYLINK DJ: Mechanical loading-induced gene expression and BMD changes are different in two inbred mouse strains. J Appl Physiol 99: 1951-1957, 2005. 
KESAVAN C, WERGEDAL JE, LAU KH, MOHAN S: Conditional disruption of IGF-I gene in type 1alpha collagenexpressing cells shows an essential role of IGF-I in skeletal anabolic response to loading. Am J Physiol Endocrinol Metab 301: E1191-E1197, 2011.

PETERS KG, COOGAN A, BERRY D, MARKS J, IGLEHART JD, KONTOS CD, RAO P, SANKAR S, TROGAN E: Expression of Tie2/Tek in breast tumour vasculature provides a new markers for evaluation of tumour angiogenesis. Br J Cancer 77: 51-56, 1998.

SAPINO A, BONGIOVANNI M, CASSONI P, RIGHI L, ARISIO R, DEAGLIO S, MALAVASI F: Expression of CD31 by cells of extensive ductal in situ and invasive carcinomas of the breast. J Pathol 194: 254-261, 2001.

SENGUL A, MOHAN S, KESAVAN C: Bone response to loading in mice with targeted disruption of the cartilage oligomeric matrix protein gene. Physiol Res 61: 637-641, 2012.

YAO Z, LAFAGE-PROUST MH, PLOUET J, BLOOMFIELD S, ALEXANDRE C, VICO L: Increase of both angiogenesis and bone mass in response to exercise depends on VEGF. J Bone Miner Res 19: 1471-1480, 2004.

ZHANG, ZJ, MA SL: miRNAs in breast cancer tumorigenesis (Review). Oncol Rep 27: 903-910, 2012.

ZHENG LW, MA L, CHEUNG LK: Angiogenesis is enhanced by continuous traction in rabbit mandibular distraction osteogenesis. J Craniomaxillofac Surg 37: 405-411, 2009. 